\title{
Evaluation and Research on Some Pilot Smart Cities
}

\author{
Fang Gong ${ }^{1 *}$ and Xueni $\mathrm{CaO}^{2}$ \\ ${ }^{1}$ Anhui Xinhua university, School of Big Data and Artificial Intelligence, Hefei 230088, China \\ ${ }^{2}$ Anhui Xinhua university, School of Big Data and Artificial Intelligence, Hefei 230088, China
}

\begin{abstract}
This paper, taking 30 pilot smart cities as the research objects, firstly presents a reasonable and comprehensive evaluation index system of smart city development from six aspects. Secondly, factor analysis method was used to reduce the dimension of 23 indicators, and 7 common factors and their weights were obtained. The total score of 30 cities' development status is ranked by the use of common factor and index data. The K-means clustering method makes cluster analysis on 30 cities, and divides each city into 4 categories according to the development situation of the indicators. Finally, based on the evaluation results, the author gives some suggestions on the construction of smart city.
\end{abstract}

\section{Introduction}

With the continuous progress of human society, the earth has been carrying more and more people. In order to support more population and provide people with a better life, many regions of the world have carried out urbanization construction. However, the acceleration and development of urbanization process have brought many problems to urban development. In order to effectively solve the dilemmas and problems faced by urban development and achieve sustainable urban development, the concept of "smart city" emerges as the times demand. All countries and regions have carried out the construction of smart cities, which has become an irreversible historical trend of urban development in today's world. Smart city development evaluation is an indispensable step in the process of smart city construction. Establishing a scientific and effective evaluation system is conducive to obtaining reliable and valuable development level information. Evaluating the development level of smart cities is conducive to urban management departments to grasp the current situation of urban development, adjust and optimize urban construction plans in time, and improve the feasibility of construction.

The construction of smart cities in China has been gradually carried out across the country. The Ministry of Housing and Urban-Rural Development, the National Development and Reform Commission, the Ministry of Industry and Information Technology and other departments have also put forward corresponding policies to guide and deploy the promotion of smart city construction. In 2013, the first batch of domestic pilot smart cities was set up, paving the way for the construction and exploration of smart cities in China. According to statistics, closed on February 2019, more than 700 cities in China have proposed or carried out the construction of smart cities, which has become a fashion trend. In the process of the construction of smart city, the factors that affect the construction of smart city have gradually become the focus of many scholars. For example, Zhang Xiekui and other scholars took smart cities in western China as an example to establish an analysis model of influencing factors from five aspects including infrastructure, economic development and industrial investment, as well as 16 secondary factors. Through quantitative analysis, the main influencing factors of smart city construction were extracted. Some scholars have also constructed a theoretical model of "factor-behavior-performance" through quantitative research. Zhang Yi and other scholars have analyzed and summarized the following three kinds of social factors that influence the success of smart city construction in China from the social perspective. This paper studies the development status of smart city to find out the key factors affecting the development of cities, and analyze the influence degree of these key factors. Through the analysis and comparison of the gap between cities, find out the characteristic competitiveness of cities, and put forward reasonable suggestions to promote the development of smart cities.

\section{The construction of index system and data preprocessing}

\subsection{The construction of index system}

Constructing a comprehensive and reasonable index system is the basis of objectively and accurately evaluating the development of smart cities. This paper constructs an evaluation index system including 6 first-level indicators and 23 second-level indicators from six aspects of smart infrastructure, smart economy, science and technology innovation driven, smart ecology, smart people's livelihood and smart population. See Table

*Email: 67482319@qq.com

(c) The Authors, published by EDP Sciences. This is an open access article distributed under the terms of the Creative Commons Attribution License 4.0 
1 for details.

Table1 Evaluation Index of Smart City Development Level

\begin{tabular}{|c|c|}
\hline Intelligent infrastructure & $\begin{array}{c}\text { Fixed Internet broadband access } \\
\text { userX1, } \\
\text { mobile phone userX2, } \\
\text { Total Business Volume of Posts and } \\
\text { TelecommunicationsX3 }\end{array}$ \\
\hline Smart Economy & $\begin{array}{c}\text { GDP per capitaX4, } \\
\text { The per capita disposable income } \\
\text { of urban residentsX5, } \\
\text { The proportion of tertiary industry } \\
\text { in GDPX6 }\end{array}$ \\
\hline $\begin{array}{l}\text { Driven by scientific and } \\
\text { technological innovation }\end{array}$ & $\begin{array}{c}\text { Scientific and technological } \\
\text { achievementsX7 } \\
\text { Number of patent applications } \\
\text { acceptedX8 } \\
\text { Number of patents grantedX9 } \\
\text { Number of research and } \\
\text { experimental development } \\
\text { enterprisesX10 } \\
\text { Ratio of expenditure to GDPX11 }\end{array}$ \\
\hline Ecological Wisdom & $\begin{array}{c}\text { Energy consumption of ten thousand } \\
\text { yuan GDPX12 } \\
\text { green coverage rate of built-up } \\
\text { areaX13 } \\
\text { Per capita park green areaX14 } \\
\text { Annual air quality levelX15 } \\
\text { urban sewage treatment rateX16 } \\
\text { Harmless treatment rate of domestic } \\
\text { garbageX17 }\end{array}$ \\
\hline $\begin{array}{l}\text { Wisdom and people's } \\
\text { livelihood }\end{array}$ & $\begin{array}{c}\text { Public Library HoldingsX18 } \\
\text { Comprehensive Population } \\
\text { Coverage of Radio and } \\
\text { TelevisionX19 } \\
\text { Public transport vehicles per 10,000 } \\
\text { peopleX20 } \\
\text { Number of beds in medical and } \\
\text { health institutionsX21 }\end{array}$ \\
\hline The wise crowd. & $\begin{array}{c}\text { Research and Experimental } \\
\text { Development StaffX22 } \\
\text { Proportion of population with higher } \\
\text { educationX23 }\end{array}$ \\
\hline
\end{tabular}

\subsection{Data reprocessing}

This paper takes 30 smart pilot cities such as Beijing, Tianjin and Harbin as the research objects, and collects the index data of these cities in the past five years. According to the relationship between the value of the index and the degree of construction, the index is divided into positive and negative indexes, and the original data is preprocessed according to Equations (1) and (2)

Positive index:

$$
X^{\prime}{ }_{i j}=\left(X_{i j}-\min X_{i j}\right) /\left(\max X_{i j}-\min X_{i j}\right)
$$

Negative index:

$$
\mathrm{X}_{\mathrm{ij}}^{\prime}=\left(\max _{\mathrm{ij}}-\mathrm{X}_{\mathrm{ij}}\right) /\left(\max \mathrm{X}_{\mathrm{ij}}-\min \mathrm{X}_{\mathrm{ij}}\right)
$$

Part of the original data and processing results are shown in Table 2.
Table 2 Part of The City Index Data

\begin{tabular}{|c|c|c|c|c|c|c|}
\hline \multirow{2}{*}{ City } & \multicolumn{3}{|c|}{ X1 } & \multicolumn{2}{c|}{ X2 } & \multicolumn{2}{c|}{ X3 } \\
\hline & before & after & before & after & before & after \\
\hline Haerbin & 393.2 & -0.10 & 1403.0 & -0.11 & 963.2 & 0.48 \\
\hline Changchun & 549.0 & 0.37 & 1092.0 & -0.43 & 243.2 & -0.53 \\
\hline Shenyang & 250.4 & -0.54 & 1273.2 & -0.24 & 766.2 & 0.20 \\
\hline Beijing & 688.5 & 0.80 & 4019.7 & 2.63 & 2759.6 & 2.99 \\
\hline Tianjin & 523.6 & 0.30 & 1704.7 & 0.21 & 1343.8 & 1.01 \\
\hline Huhehaote & 256.1 & -0.53 & 459.3 & -1.10 & 203.3 & -0.58 \\
\hline
\end{tabular}

\section{Factor analysis}

Factor analysis method is a multivariate statistical analysis method that simplifies some variables with complex relations into a few comprehensive factors, starting from the study of the internal dependence of Variable Correlation Matrix.

\subsection{Variable correlation test}

KMO and Bartlett tests were carried out to check whether the partial correlation between variables was strong enough and to determine whether the correlation matrix was the Identity Matrix. According to Table 3, the KMO value is 0.717 , which indicates that the effect of factor analysis and the amount of information overlap among variables is acceptable. Bartlett test shows that there is a strong correlation between the variables, which can be analyzed in the next step.

Table $3 \mathrm{KMO}$ and Bartlett's Test

\begin{tabular}{|l|l|r|}
\hline \multicolumn{2}{|l|}{$\begin{array}{l}\text { Kaiser-Meyer-Olkin Measure of Sampling } \\
\text { Adequacy. }\end{array}$} & .717 \\
\hline \multirow{3}{*}{$\begin{array}{l}\text { Bartlett's Test of } \\
\text { Sphericity }\end{array}$} & Approx. Chi-Square & 2607.067 \\
\cline { 2 - 3 } & df & 253 \\
\cline { 2 - 3 } & Sig. & .000 \\
\hline
\end{tabular}

\subsection{Extraction of common factors}

The number of principal component eigenvalues indicates how many original variables it carries. The factor contribution rate indicates the ratio of the factor to all principal component variances. The first seven principal components are used to describe the overall level of urban development instead of all the variables, because the cumulative contribution rate of variance is $74.951 \%$ in table 4 , which conforms to the selection principle. 
Table 4 Total Variance Explained

\begin{tabular}{|c|c|c|c|c|c|c|}
\hline \multirow{2}{*}{$\begin{array}{c}\text { comp } \\
\text { onent }\end{array}$} & \multicolumn{3}{|c|}{ Initial eigenvallues } & \multicolumn{3}{c|}{$\begin{array}{c}\text { Rotation Sums of Squared } \\
\text { Loadings }\end{array}$} \\
\cline { 2 - 7 } & Total & $\begin{array}{c}\text { \% of } \\
\text { Variance }\end{array}$ & Cumulative \% & Total & $\begin{array}{c}\% \text { of } \\
\text { Variance }\end{array}$ & Cumulative \% \\
\hline 1 & 7.143 & 31.058 & 31.058 & 6.132 & 26.662 & 26.662 \\
\hline 2 & 2.452 & 10.659 & 41.717 & 2.424 & 10.540 & 37.202 \\
\hline 3 & 1.830 & 7.955 & 49.672 & 1.839 & 7.996 & 45.198 \\
\hline 4 & 1.709 & 7.433 & 57.105 & 1.797 & 7.813 & 53.011 \\
\hline 5 & 1.560 & 6.783 & 63.888 & 1.703 & 7.402 & 60.413 \\
\hline 6 & 1.442 & 6.269 & 70.157 & 1.702 & 7.402 & 67.815 \\
\hline 7 & 1.103 & 4.794 & 74.951 & 1.641 & 7.136 & 74.951 \\
\hline
\end{tabular}

The seven common factors were named F1 F7.

\subsection{Calculate the overall score of each city}

First, the regression algorithm was used to calculate the scoring coefficient of each common factor, as shown in Table 5.

Table 5 Component Matrix

\begin{tabular}{|l|r|r|r|r|r|r|r|}
\hline & \multicolumn{7}{|c|}{ Component } \\
\cline { 2 - 8 } & \multicolumn{1}{|c|}{1} & \multicolumn{1}{c|}{2} & \multicolumn{1}{c|}{3} & \multicolumn{1}{c|}{4} & \multicolumn{1}{c|}{5} & \multicolumn{1}{c|}{6} & \multicolumn{1}{c|}{7} \\
\hline $\mathrm{X} 1$ & -.080 & .397 & -.008 & -.045 & .076 & -.040 & .192 \\
\hline $\mathrm{X} 2$ & .075 & .198 & -.009 & -.067 & .054 & -.013 & -.101 \\
\hline $\mathrm{X} 3$ & .169 & -.007 & -.107 & -.102 & .108 & -.026 & .052 \\
\hline $\mathrm{X} 4$ & .139 & -.163 & .068 & .052 & -.062 & -.010 & .175 \\
\hline $\mathrm{X} 5$ & .146 & -.124 & .078 & .069 & -.081 & .066 & -.009 \\
\hline $\mathrm{X} 6$ & .120 & .025 & -.168 & .111 & .199 & .019 & -.134 \\
\hline $\mathrm{X} 8$ & .062 & -.089 & -.167 & .135 & -.251 & -.203 & -.169 \\
\hline $\mathrm{X} 9$ & .149 & .000 & -.014 & .006 & -.028 & .011 & -.008 \\
\hline $\mathrm{X} 10$ & .052 & -.005 & -.017 & -.024 & -.030 & .017 & -.024 \\
\hline $\mathrm{X} 11$ & -.001 & -.031 & .076 & -.123 & -.292 & .101 & .146 \\
\hline $\mathrm{X} 12$ & -.055 & .315 & .059 & -.006 & -.147 & .311 & .048 \\
\hline $\mathrm{X} 13$ & -.021 & -.050 & .427 & .105 & .121 & -.089 & -.018 \\
\hline $\mathrm{X} 14$ & .029 & -.007 & .089 & -.048 & .504 & .009 & -.054 \\
\hline $\mathrm{X} 15$ & .111 & -.110 & .134 & -.333 & .011 & .286 & -.251 \\
\hline $\mathrm{X} 16$ & -.016 & .055 & -.102 & .055 & -.035 & -.068 & .498 \\
\hline $\mathrm{X} 17$ & -.084 & .077 & .507 & -.063 & -.006 & .020 & -.130 \\
\hline $\mathrm{X} 18$ & .136 & -.020 & -.005 & .081 & -.034 & .027 & -.228 \\
\hline $\mathrm{X} 19$ & .012 & -.120 & .106 & .019 & -.045 & -.408 & .158 \\
\hline $\mathrm{X} 20$ & .024 & -.024 & -.037 & .115 & -.055 & .433 & .027 \\
\hline $\mathrm{X} 21$ & -.071 & .385 & .034 & .023 & -.028 & .031 & -.083 \\
\hline $\mathrm{X} 22$ & .162 & -.071 & -.179 & -.155 & .197 & -.137 & .230 \\
\hline $\mathrm{X} 23$ & .004 & -.030 & -.094 & .349 & .182 & .148 & .225 \\
\hline & & & & & & & \\
\hline
\end{tabular}

Then according to the variance contribution rate of each common factor after rotation in Table 4, the weight of each common factor is $0.356,0.141,0.107,0.104$, $0.099,0.099$ and 0.095 .

The calculation formula for the total score of each city is as follows:

Score $=0.356 * \mathrm{~F} 1+0.141 * \mathrm{~F} 2+0.107 * \mathrm{~F} 3+$ $0.104 * \mathrm{~F} 4+0.099 * \mathrm{~F} 5+0.099 * \mathrm{~F} 6+0.095 * \mathrm{~F} 7$

$$
\mathrm{Fj}=\sum_{i=1}^{23} a_{i j} * \mu_{i}, \quad \mathrm{j}=1 \sim 7
$$

$a_{i j}$ is the scoring coefficient of the jth common factor on the ith index, and $u_{i}$ is the value of the city on the ith index.

The total scores and rankings of the 30 cities were calculated, as shown in Table 6

Table 6 City Scores And Rankings

\begin{tabular}{|c|c|c|c|c|c|}
\hline City & Score & Rank & City & Score & Rank \\
\hline Beijing & 1.36 & 1 & Ningbo & 0.06 & 17 \\
\hline Suzhou & 1.07 & 4 & Zhengzhou & 0.4 & 10 \\
\hline Shenzhen & 1.07 & 3 & Huhehaote & -0.12 & 21 \\
\hline Guangzhou & 1.23 & 2 & Shenyang & -0.13 & 22 \\
\hline Nanjing & 0.55 & 6 & Nanchang & -0.25 & 25 \\
\hline Shanghai & 0.72 & 5 & Foshan & 0.02 & 19 \\
\hline Hangzhou & 0.52 & 7 & Changsha & 0.15 & 15 \\
\hline Wuxi & 0.45 & 9 & Guiyang & -0.07 & 20 \\
\hline Chengdu & 0.47 & 8 & Fuzhou & -0.18 & 24 \\
\hline Hefei & 0.35 & 11 & Wuhu & -0.26 & 26 \\
\hline Jinan & 0.06 & 18 & Wenzhou & -0.15 & 23 \\
\hline Chongqing & 0.23 & 12 & Tianjin & -0.32 & 28 \\
\hline Changchun & -0.3 & 27 & Lanzhou & 0.21 & 13 \\
\hline Qingdao & 0.17 & 14 & Haerbin & -0.42 & 29 \\
\hline Wuhan & 0.14 & 16 & Yinchuan & -0.68 & 30 \\
\hline
\end{tabular}

\subsection{Regional ranking}

The 30 cities are divided into 7 regions according to their geographical location. The score for the development level of each region is the average score for all the cities in the region. The specific results are shown in Table 7.

South China has maintained its leading position in the past three years, and its scores have increased greatly every year. The gap between North China, East China, Southwest China and Central China is narrowing year by year. In general, the scores of each region have made great progress in the past three years, and the gap between regions is getting smaller and smaller. The 
number of regions with positive scores is increasing gradually. Especially in the past two years, the development of various regions is particularly rapid. The construction of smart cities in all regions is proceeding in an orderly way, forming a favorable situation.

Table 7 Region Scores And Rankings

\begin{tabular}{|c|c|c|c|c|c|c|}
\hline \multirow{2}{*}{ Region } & \multicolumn{2}{|c|}{2017} & \multicolumn{2}{c|}{2018} & \multicolumn{2}{c|}{2019} \\
\cline { 2 - 7 } & score & Ranking & score & Ranking & score & Ranking \\
\hline South China & 0.34 & 1 & 0.55 & 1 & 0.77 & 1 \\
\hline north China & 0.08 & 2 & 0.23 & 2 & 0.31 & 2 \\
\hline East China & 0.02 & 4 & 0.12 & 3 & 0.24 & 3 \\
\hline Southwest & 0.05 & 3 & 0.12 & 4 & 0.21 & 5 \\
\hline Central China & -0.07 & 5 & 0.06 & 5 & 0.23 & 3 \\
\hline Northeast & -0.37 & 6 & -0.21 & 6 & -0.24 & 6 \\
\hline Northwest & -0.53 & 7 & -0.29 & 7 & -0.28 & 7 \\
\hline
\end{tabular}

\section{Clustering analysis}

K-means clustering algorithm is an iterative solution of the cluster analysis algorithm. It uses distance as a similarity metric to find $\mathrm{K}$ classes in a given data set. The center of each class is obtained according to the mean value of all the values in the class, and the center of each class is described by the cluster center. Clustering centers and the objects assigned to them represent a cluster. For each sample allocated, the cluster center of the cluster will be recalculated based on the existing objects in the cluster. This process does not stop until some termination condition is met. The specific steps are as follows:

Input: Sample set $\mathrm{D}=\left\{x_{1}, x_{2}, \ldots x_{m}\right\}, \mathrm{K}$ is the number of clusters in the cluster, $\mathrm{N}$ is the maximum number of iterations.

Output: Cluster partition $\mathrm{C}=\left\{C_{1}, C_{2}, \ldots C_{k}\right\}$

1) Randomly select $k$ samples from sample set $D$ as the initial k centroid vectors: $\left\{u_{1}, u_{2}, \ldots u_{k}\right\}$

2) For $\mathrm{n}=1,2 \ldots, \mathrm{N}$

a) Initialize the cluster partition $\mathrm{C}$ as $C_{t}=\emptyset t=$ $1,2 \ldots k$

b) Calculate the distance $d_{i j}$ between the sample $x_{i}$ and each centroid vector $u_{j}(j=$ $1,2 \ldots k)$

$d_{i j}=\left\|x_{i}-u_{j}\right\|_{2}^{2}$ For $\mathrm{i}=1,2 \ldots \mathrm{m}$

The smallest mark of $x_{i}$ is the class $\delta_{i}$ corresponding to $D_{i j}$.

Update $C_{\delta_{i}}=C_{\delta_{i}} \cup\left\{x_{i}\right\}$

c) The new center $u_{j}$ of mass is recalculated for all sample points in $C_{j}$.

$u_{j}=\frac{1}{\left|C_{j}\right|} \sum_{x \in C_{j}} x$, For $\mathrm{j}=1,2 \ldots \mathrm{k}$

d) If all $\mathrm{k}$ centroid vectors have not changed, go to step 3)

3) Output cluster partition $\mathrm{C}=\left\{C_{1}, C_{2}, \ldots C_{k}\right\}$

The 30 cities were classified into 4 categories by
K-means clustering according to the scores of each common factor. The specific classification of cities is shown in Table 7.

Table 8 City Clustering

\begin{tabular}{|c|c|c|c|c|}
\hline cluster & 1 & 2 & 3 & 4 \\
\hline Num & 5 & 10 & 13 & 2 \\
\hline & & & Fuzhou & \\
& & Changchun & Guangzhou & \\
& & Huhehaote & \\
& & Changsha & Haerbin & \\
& Suzhou & Chengdu & Nanchang & \\
& Shanghai & Foshan & Ningbo & Guiyang \\
& Nanjing & Hefei & Qingdao & Lanzhou \\
& Hangzhou & Wuxi & Shenzhen & \\
& Beijing & Wuhan & Shenyang & \\
& & Tianjin & \\
& & Zhengzhou & Wenzhou、 & \\
& & Chongqing & Wuhu & \\
& & & Yinchuan & \\
\hline
\end{tabular}

The total scores of the four categories obtained by cluster analysis are basically decreasing. Each category has its own advantages and disadvantages in the evaluation index. The first kind of cities performs well in the comprehensive level of urban life and the development level of urban science and technology, but they do not perform well in the level of urban sewage treatment. The total score of the second type of cities is excellent in infrastructure, but poor in urban planning. The third kind of cities perform well in comprehensive living standard and urban planning development level, but not well in urban science and technology development level and urban traffic culture level. The fourth kind of cities performs well in urban planning, urban traffic culture and urban sewage treatment, but they do not perform well in the comprehensive level of urban living and urban environmental protection.

\section{Conclusion}

The construction of smart city in our country is still in the exploration stage, the construction of smart city is not mature, there are still big differences in the development among regions, and the construction of each city in the construction process cannot be well coordinated in all aspects.

Shenzhen performed best in terms of overall urban living standards. Chongqing performed best in terms of the level of urban infrastructure. Foshan topped the list in terms of urban environmental protection. Beijing performed best in terms of the level of urban science and technology development. Guangzhou performed best in terms of the level of urban planning and development. Lanzhou is the most outstanding city in terms of the development level of urban transportation culture. Zhengzhou performs best in the aspect of urban transportation literacy.

1) Building a sound SMART infrastructure. Perfect and good infrastructure is an important guarantee for the success of urban construction. 
2) Implementing the strategy of innovation-driven development. Innovation is the vitality of a smart city. Urban construction can be faster and stronger only by enhancing the innovation capacity of cities.

3) Formulating urban development program which reflects the characteristics of the city and adapts to local conditions;

4) Strengthening inter-city cooperation and jointly building regional economic integration.

\section{Acknowledgements}

The authors would like to thank the anonymous reviewers for their valuable suggestions as to how to improve this paper. This work was supported by the social Science Foundation of the Anhui Educational Committee of China (Grant Nos. Sk2017A0684)

\section{References}

1. Zhang,XK. (2016) Study on Influencing Factors of Smart City Construction in Western China. Ecological Economy, 32:110-115.
2. Cui,Qh.(2017)Action Mechanism Of Influential Factors On Objective Performances In Smart City Construction. Journal Of Tongji University (Natural, Science),45:152-158.

3. Hu,N.(2019)Research On Evaluation Model Of Smart City Development Level. Dalian Maritime University.

4. Jiao, LF. (2015)Research on China's Smart City Construction and Government Management. Xi 'an University of Architecture and Technology.

5. Ren,1.(2019)Research On The Evaluation Of Development Level Of Smart City Based On Entropy TOPSIS Model. Information Studies: Theory\&Application,42:113-118+125.

6. Zuo, MT.(2019) Study on evaluation of development level of smart city,06:16-17.

7. Xu,jw.(2019)Evaluation Of Development Of Smart Cities In Jiangsu Province Based On Principal Component Analysis. Construction Science And Technology,15:70-75. 